Canadian

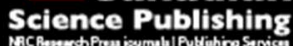

Applied Physiology, Nutrition, and Metabolism Physiologie appliquée, nutrition et métabolisme

\title{
Non-localized postactivation performance enhancement (PAPE) effects in trained athletes: a pilot study
}

\begin{tabular}{|r|l|}
\hline Journal: & Applied Physiology, Nutrition, and Metabolism \\
\hline Manuscript ID & apnm-2017-0217.R1 \\
\hline Manuscript Type: & Brief communication \\
\hline Date Submitted by the Author: & 10 -Jun-2017 \\
\hline Complete List of Authors: & $\begin{array}{l}\text { Cuenca-Fernández, Francisco; Universidad de Granada Facultad de } \\
\text { Ciencias de la Actividad Fisica y del Deporte } \\
\text { Smith, Ian; University of Calgary, Human Performance Lab } \\
\text { Jordan, Matthew; University of Calgary, Human Performance Lab } \\
\text { MacIntosh, Brian; University of Calgary, Human Performance Lab } \\
\text { López-Contreras, Gracia; Universidad de Granada Facultad de Ciencias de } \\
\text { la Actividad Fisica y del Deporte } \\
\text { Arellano, Raúl; Universidad de Granada Facultad de Ciencias de la } \\
\text { Actividad Fisica y del Deporte } \\
\text { Herzog, Walter; University of Calgary, Human Performance Lab }\end{array}$ \\
\hline Is the invited manuscript for \\
consideration in a Special \\
Issue? :
\end{tabular}

\section{SCHOLARONE"}

Manuscripts 
1 Non-localized postactivation performance enhancement (PAPE) effects in trained athletes: a pilot study

2 Cuenca-Fernández, Francisco ${ }^{1^{*}}$; Smith, Ian $\mathrm{C}^{2 *}$; Jordan, Matthew $\mathrm{J}^{2}$; MacIntosh, Brian $\mathrm{R}^{2}$; López-Contreras,

3 Gracia $^{1}$; Arellano, Raúl ${ }^{1}$; Herzog, Walter $^{2}$

*- These authors contributed equally to this work.

5

†Corresponding Author: icsmith@ucalgary.ca

1- Department of Physical Education and Sport, Faculty of Sport Sciences. University of Granada, Granada, Spain

Faculty of Sport Sciences

Carretera de Alfacar, sn

University of Granada

18011-Granada

Spain

2-Human Performance Laboratory. Faculty of Kinesiology. University of Calgary, Calgary, Canada.

16

17

18

19

20

21

22

Human Performance Lab

University of Calgary

2500 University Dr NW

Calgary, Alberta, Canada

T2N $1 \mathrm{~N} 4$ 
24 Abstract: jumps (SJ) and power push-ups (PPU) following upper body activation, lower body activation, upper and lower

27 body activation, and rest. SJ improved similarly across all four conditions. PPU could not be assessed. Since the test protocol of SJ and PPU involved upper and lower body activation and caused PAPE in SJ, future work is required to determine if a non-localized PAPE effect exists.

30 Key words: squat jump, postactivation potentiation, voluntary contractions, muscle function, swimming 


\section{Introduction:}

The athletic community has shown considerable interest in the performance enhancements seen soon after a warm-up of brief, high force contractions (conditioning activity; CA) (Sale 2004). The magnitude and time history of the enhancement depends on whether performance is assessed in electrically evoked or voluntary contractions. Enhancements of electrically evoked contractions are typically large $(>20 \%)$ increases in twitch torque during the first minute after the CA and decline rapidly (Tillin and Bishop 2009; Vandenboom 2016). In contrast, enhancements of voluntary contractions are typically small $(<5 \%)$ effects observable after a significant rest period, peaking 7-10 minutes after the CA (Maloney et al. 2014; Tillin and Bishop 2009; Wilson et al. 2013), a time when there is effectively no remaining enhancement of electrically evoked contractions. The differences between these effects have been obscured by imprecise use of terminology in the literature. The term postactivation potentiation (PAP) classically refers to enhancement of electrically evoked twitch force (Belanger et al. 1983; Vandervoort et al. 1983). This definition has not been strictly adhered to, with several papers purportedly studying PAP having only measured enhancement of voluntary activations. In this paper we refer to the enhancement of electrically evoked contractions as PAP, and the enhancement of voluntary movements as postactivation performance enhancements (PAPE).

There is strong evidence that PAP is a local effect caused by contraction-induced increases in myosin regulatory light chain (RLC) phosphorylation (Vandenboom 2016). PAPE, however, could be achieved via a number of effects unrelated to RLC phosphorylation including increased muscle temperature (MacIntosh et al. 2012; McGowan et al. 2015; Sargeant 1987), increased recruitment of motor units (Tillin and Bishop 2009), and increased excitability or firing synchrony of motor neurons (Güllich and Schmidtbleicher 1996; Trimble and Harp 1998; Vandenboom 2016). The inotropic effects of exercise-induced elevations in plasma catecholamines (Cairns and Borrani 2015; Decostre et al. 2000) may also contribute to PAPE, but this has not been investigated in detail. Specifically, brief bouts of intense exercise can increase circulating epinephrine and norepinephrine levels (Botcazou et al. 2006). Exposure to these catecholamines enhances force in both fast and slow muscle fibres (Cairns and Dulhunty 1993). As circulating hormones, norepinephrine and epinephrine could systemically enhance muscle contraction. A non-localized PAPE effect is an intriguing notion, however, we are not aware of any study which has tested for PAPE in muscle groups which were not activated by the CA. Such an effect would be of great interest to the sporting world as it could circumvent the detrimental effects of neuromuscular fatigue (Pierce 1995). In this study we assessed squat jump (SJ) and power push-up (PPU) performance in 
trained swimmers before and after four different CAs: quiet standing (QS), back squat (BS), bench press (BP), and BS+BP. We hypothesized that there would be no PAPE following QS, and chose the conservative hypothesis that PAPE effects would be purely local responses.

\section{Materials and methods:}

\section{Subjects}

Fifteen varsity level swimmers ( 8 males and 7 females) volunteered to participate in this study (mean \pm SD, Age: $19.4 \pm 1.4$ years, Weight: $78.6 \pm 9.0 \mathrm{~kg}$ [males], $65.4 \pm 8.5 \mathrm{~kg}$ [females], Height: $1.83 \pm 0.02 \mathrm{~m}$ [males], $1.64 \pm 0.06 \mathrm{~m}$ [females]). Swimmers were in their competition period and had participated in national and international competitions for at least 1 year prior to the start of the study. The swimmers habitually trained 6 days per week using a complex training protocol which allowed the development of power and speed while decreasing the volume of aerobic training (Hydren and Cohen 2015). None of the swimmers were taking drugs, medication, or dietary supplements known to influence physical performance. Tests took place prior to their daily training regimen, and subjects were instructed to avoid any physical exertion prior to testing. Each test day began with participants standing quietly for 10 minutes. Test familiarization was performed during their dry practices held three times per week. The loads required to perform 1 repetition maximum (1RM) back squat and bench press lifts were determined during the familiarization period. The $1 \mathrm{RM}(\operatorname{mean} \pm \mathrm{SD})$ for back squat was $90.7 \pm 17.0 \mathrm{~kg}$ for males and $53.1 \pm 14.1 \mathrm{~kg}$ for females, and the $1 \mathrm{RM}$ for bench press was $71.3 \pm 12.2 \mathrm{~kg}$ for males and $34.1 \pm 10.3 \mathrm{~kg}$ for females. All experiments were performed in the Olympic Oval at the University of Calgary. Subjects signed an informed consent form which was reviewed and approved by the Conjoint Health Research Ethics Board at the University of Calgary (REB 15-1135).

\section{Experimental approach}

A repeated measures counterbalanced design was used in which swimmers were evaluated for SJ and PPU performance before and after each of four different CAs tested over four different days. The BS CA consisted of four BS repetitions at 90\% 1RM, the BP CA consisted of four BP repetitions at 90\% 1RM, and the $\mathrm{BS}+\mathrm{BP}$ CA consisted of four BS and four BP repetitions, each at 90\% 1RM. The QS CA served as a control 
condition in which participants were instructed to stand quietly for four minutes, equal to the time required to perform the heavy resistance exercises. SJ and PPU performances were assessed four minutes prior to (Pre), and at 5, 8, 12 and 20 minutes following (Post-5, Post-8, Post-12, and Post-20) the completion of each CA. To permit consideration of inter-individual differences in the timing of PAPE effects, the highest impulse generated during Post measures was designated Post-Max. This study was designed in accordance with the schematic guidelines established in MacIntosh et al. (2012) for determining the impact of warm-up activities on athletic performance

Each PPU and SJ movement began with subjects performing a countermovement followed by a 2 second static hold prior to initiating the ballistic movements. SJ were performed with both feet on the force plate at takeoff and landing, hands placed on the hips. Squat depth was self-selected. The PPU were performed with both hands on the force plate during push off and landing. The body position during the static hold was selfselected. Toes remained in contact with the ground at all times. Six of the seven females performed a modified PPU in which both knees and toes maintained contact with the ground. An encouraging verbal signal was given as the start command for each ballistic movement. Performance was assessed from the ground reaction force (GRF) exerted on the force plate.

\section{Impulse analysis}

Muscle performance was inferred from the vertical impulse obtained from the GRF vs time recording from a force plate (PASCO ${ }^{\circledR}$, PS-2141. Roseville, CA 95747 USA). Data were collected at $1000 \mathrm{~Hz}$ using DataStudio (version 1.9.8r10). Impulse was calculated using the impulse-momentum method shown in equation 1 (Linthorne 2001).

Jump Impulse $=\int_{t_{I}}^{t_{\mathrm{TO}}}\left(F_{\mathrm{GRF}}-m \cdot g\right) \cdot d t$

Here, the impulse associated with the lower limb musculature is equal to the integration of the GRF-time record from the time of jump initiation $\left(t_{I}\right)$ to time of take-off $\left(t_{\mathrm{TO}}\right)$ minus the product of body weight and the acceleration due to gravity, adjusted for elevation $(m \bullet g$; a constant value for each subject each test day) over this same time period. For practical considerations, the $m \bullet g$ term was determined from the GRF during a period of quiet standing each collection day. The $t_{I}$ was typically assigned to be the final instant at which the GRF dropped below $m \cdot g$ prior to the jump. However, if this method of finding $t_{I}$ placed $t_{I}$ more than $1 \mathrm{~s}$ prior to the jump, $t_{I}$ was set to be the time at which the GRF was closest to $m \cdot g$ within $1 \mathrm{~s}$ prior to the jump. The time the 
117 GRF reached zero during the jump was designated $t_{\mathrm{TO}}$. Data are presented as the best performance out of the 3

118 SJ performed at each time point. In the PPU task, the proportion of body mass supported by the arms changed as 119 the upper body was raised and lowered. The instability of the effective $m \cdot g$ term for the PPU reduced our confidence in our ability to accurately identify PAPE effects in this task. Accordingly, PPU performance is not considered further in this manuscript.

\section{Statistical analysis} for normality of distribution, 2-way (time x condition) repeated measures ANOVAs were used to compare impulse at Pre-Post time points. Comparisons between Pre and Post-Max were assessed with a 2-way (time $\mathrm{x}$ condition) repeated measures ANOVA. Tukey’s honest significant difference (HSD) post hoc test was used to obtain specific comparisons when warranted. Differences were considered significant at $\alpha<0.05$. All values are reported as mean $\pm \mathrm{SD}$. Males and females were grouped together in all analyses.

\section{Results:}

The coefficient of variation in SJ performance across all Pre trials was 0.060 . There were no significant systematic differences in best jump performance at Pre between days (Figure 1A). No significant differences in impulse were observed between the different times and conditions when all 5 time points were considered. To increase our statistical power, Post-12 and Post-20 were omitted from the analysis, resulting in no significant interaction but a significant main effect of time. Post hoc testing revealed a significant increase $(P<0.05)$ in jump impulse at Post-5 relative to Pre (Figure 1B). Post-Max was significantly greater $(P<0.05)$ than Pre as a main effect of a 2 way repeated measures ANOVA (not depicted). The percent change in impulse between Pre and Post-5 and between Pre and Post-Max were compared across the four CAs (Figure 1C) using 1 way repeated measures ANOVAs. In these analyses, no statistically significant differences were seen. To determine

140 if inter-day differences in performance at Pre may have influenced PAPE, we correlated the Pre-Post changes in

141 impulse with performance at Pre relative to the four day average performance at Pre (Figure 1D). This analysis

142 revealed a significant $(P<0.001)$ inverse relationship between the two variables such that a relatively strong performance at Pre would tend to decrease the likelihood of seeing PAPE on that particular day.

\section{Discussion:}


We aimed to determine if PAPE effects could be elicited when the CA and test activity activated different groups of muscles. We found PAPE effects during SJ in a group of trained swimmers at Post-5 in all

147 CAs tested, including the QS condition. Since the appearance of PAPE is highly dependent on the individual being tested we also examined Post-Max, and again found jump impulse significantly improved relative to Pre but did not differ between CAs. The PAPE we observed is consistent in timing and magnitude to that reported by other studies (reviewed in Hodgson et al. 2005; Maloney et al. 2014; Wilson et al. 2013). Interestingly, the higher demands of the BS+BP condition did not detract from SJ performance. The PAPE found following the QS CA was a surprising result which suggests that the modest performance enhancements seen in this study were a warm-up effect, probably caused by the SJ and PPU test protocol itself. The combination of upper and lower body activity in our test activity and the PAPE effect in the QS condition prevents a conclusion favoring either the presence or absence of a non-localized PAPE effect. This remains an important question in PAPE research which could be addressed using a modification of the current study design, focusing on the performance of a single test activity before and after a similar series of CAs. We also recommend a long delay between the pre-test and the CA to avoid potential warm-up effects from the pre-test. Given the uncertainty regarding the cause of PAPE, examination of electrically evoked twitch characteristics and electromyography also seem warranted in future work to differentiate between enhanced contractility and enhanced activation. negative correlation between how well our participants performance at Pre relative to the other test days and PAPE effects in SJ. This finding has implications for the testing of individual athletes, where an exemplar Pre performance would decrease the likelihood of seeing PAPE, and vice versa. This variability should be accounted for when assessing the effectiveness of a particular warm-up procedure. Future studies could benefit from the use of a large number of Pre tests to differentiate normal variability in performance from true PAPE effects. significant, Post-Max impulse was lower in BP and BS+BP than in QS and BS, and there were performance reductions at some Post time points relative to Pre seen in BP and BS+BP, but not in QS or BS. By extension it seems possible that high-level activation of non-specific muscle groups may impair performance. The

171 implications of this interpretation further highlight the need to revisit the possible existence of non-localized PAPE effects using more sensitive and sport-specific tests. 

three PPU offered equivalent SJ performance benefits as the three heavy resistance exercise CAs. The acute

175 beneficial effect of these callisthenic exercises on performance has the advantage of requiring no specialized equipment, facilitating their use pool-side during competitions should they also offer sport-specific benefits. in electrically evoked contractions (PAP).

180 The authors report no conflicts of interest associated with this manuscript.

\section{Acknowledgements:}

182 This project DEP 2014-59707-P "SWIM: Specific Water Innovative Measurements applied to the development 183 of International Swimmers in Short Swimming Events (50 and 100M)" was financed by the Spanish Ministry of 184 Economy, Industry and Competitiveness (Spanish Agency of Research) and European Regional Development 185 Fund. Funding was also provided by Alberta Innovates, Canada Research Chair Programme, Canadian Institutes 186 of Health Research, Natural Sciences and Engineering Research Council of Canada, and the Killam Foundation.

187 This article is part of an international thesis belonging to the Program of $\mathrm{PhD}$ in Biomedicine (B11.56.1), from 188 the University of Granada, Granada, Spain. 
References

Belanger, A.Y., McComas, A.J., Elder, G.B.C. 1983. Physiological properties of two antagonistic human muscle groups. Eur. J. Appl. Physiol. 51: 381-393. doi:10.1007/BF00429075. PMID:6685037.

Botcazou, M., Gratas-Delamarche, A., Allain, S., Jacob, C., Bentué-Ferrer, D., Delamarche, P., Zouhal, H. 2006. Influence de la phase du cycle menstruel sur les réponses en catécholamines à l'exercice de sprint chez la femme. Appl. Physiol. Nutr. Metab. 31(5): 604-611. doi:10.1139/H06038. PMID:17111015.

Cairns, S.P., Borrani, F. 2015. $\beta$-adrenergic modulation of skeletal muscle contraction: key role of excitation-contraction coupling. J. Physiol. 593(21): 4713-4727. doi:10.1113/JP270909. PMID:26400207.

Cairns, S.P., Dulhunty, A.F. 1993. The effects of beta-adrenoceptor activation on contraction in isolated fast- and slow-twitch skeletal muscle fibres of the rat. Br. J. Pharmacol. 110(3): 11331141. doi:10.1111/j.1476-5381.1993.tb13932.x. PMID:8298802.

Decostre, V., Gillis, J.M., Gailly, P. 2000. Effect of adrenaline on the post-tetanic potentiation in mouse skeletal muscle. J. Muscle Res. Cell Motil. 21(247): 254. doi:10.1023/A:1005685900196. PMID:10952172.

Güllich, A., Schmidtbleicher, D. 1996. MVC-induced short-term potentiation of explosive force. New Stud. Athlet. 4: 67-80.

Hodgson, M., Docherty, M., Robbins, D. 2005. Post-activation potentiation: underlying physiology and implications for motor performance. Sports Med. 35(7): 585-595. doi: 0112-1642/05/00070585/\$34.95/0. PMID:16026172. 
Hydren, J.R., Cohen, B.S. 2015. Current scientific evidence for a polarized cardiovascular endurance training model. J. Strength Cond. Res. 29(12): 3523-3530. doi:10.1519/JSC.0000000000001197. PMID:26595137.

Linthorne, N.P. 2001. Analysis of standing vertical jumps using a force platform. Am. J. Phys. 69: 1198-1204. doi:10.1119/1.1397460.

MacIntosh, B.R., Robillard, M.E., Tomaras, E.K. 2012. Should postactivation potentiation be the goal of your warm-up? Appl. Physiol. Nutr. Metab. 37: 546-550. doi:10.1139/h2012-016. PMID:22515147

Maloney, S.J., Turner, A.N., Fletcher, I.M. 2014. Ballistic exercise as a pre-activation stimulus: a review of the literature and practical applications. Sports Med. 44(10): 1347-1359. doi:10.1007/s40279-014-0214-6. PMID:24943044.

McGowan, C.J., Pyne, D.B., Thompson, K.G., Rattray, B. 2015. Warm-up strategies for sport and exercise: mechanisms and applications. Sports Med. 45(11): 1523-1546. doi:10.1007/s40279-0150376-x. PMID:26400696.

Pierce, P. A. 1995. Fatigue: neural and muscular mechanisms, Springer. New York, NY.

Sale, D. 2004. Postactivation potentiation: role in performance. Br. J. Sports Med. 38: 386-387. doi:10.1136/bjsm.2002.003392.PMID:15273166.

Sargeant, A.J. 1987. Effect of muscle temperature on leg extension force and short-term power output in humans. Eur. J. Appl. Physiol. Occup. Physiol. 56(6): 693-698. doi:10.1007/BF00424812. PMID:3678224.

Tillin, N.A., Bishop, D. 2009. Factors modulating post-activation potentiation and its effect on performance of subsequent explosive activities. Sports Med. 29(2): 147-166. doi:10.2165/00007256-200939020-00004. PMID:19203135. 
11

236 Trimble, M.H., Harp, S.S. 1998. Postexercise potentiation of the H-reflex in humans. Med. Sci. Sports 237 Exerc. 30(6): 933-941. doi:10.1097/00005768-199806000-00024. PMID:9624654.

238 Vandenboom, R. 2016. Modulation of skeletal muscle contraction by myosin phosphorylation.

239 Compr. Physiol. 7: 171-212. doi:10.1002/cphy.c150044. PMID:28135003.

240 Vandervoort, A.A., Quinlan, J., McComas, A.J. 1983. Twitch potentiation after voluntary contraction.

241 Exp. Neurol. 81: 141-152. doi: 10.1016/0014-4886(83)90163-2. PMID:6861942.

242 Wilson, J.M., Duncan, N.M., Marin, P.J., Brown, L.E., Loenneke, J.P., Wilson, S.M.C., Jo, E.,

243 Lowery, R.P., Ungrinowitsch, C. 2013. Meta-analysis of postactivation potentiation and power:

244 effects of conditioning activity, volume, gender, rest periods and training status. J. Strength Cond.

245 Res. 27(3): 854-859. doi:10.1519/JSC.0b013e31825c2bdb. PMID:22580978. 
12

246

247

248

249

250

251

252

253

254

\section{Figure Legend}

Figure 1: Squat jump performance during Pre and Post trials. A) Mean performance in Pre trials across the four test days (no significant differences). B) Squat jump impulse at Pre, Post- 5 and Post 8 in the four test conditions. ${ }^{*} P<0.05$ vs Pre (Main effect of the 2-way repeated measures ANOVA). C) Pre-Post changes in impulse at Post-5 and Post-Max for each conditioning activity (no significant differences). D) Scatter plot of Pre-Post change in impulse at Post-5 and Post-Max versus the ratio of same-day Pre to the four day average of Pre values. Linear fits of the data have $r^{2}$ values of 0.20 for Post-5 and 0.19 for Post-Max. Both slopes are significantly different from zero with $P<0.001$. In A-C, values are presented as means \pm SD; $n=15$. QS - Quiet Standing; BS - Back Squat; BP - Bench Press. 

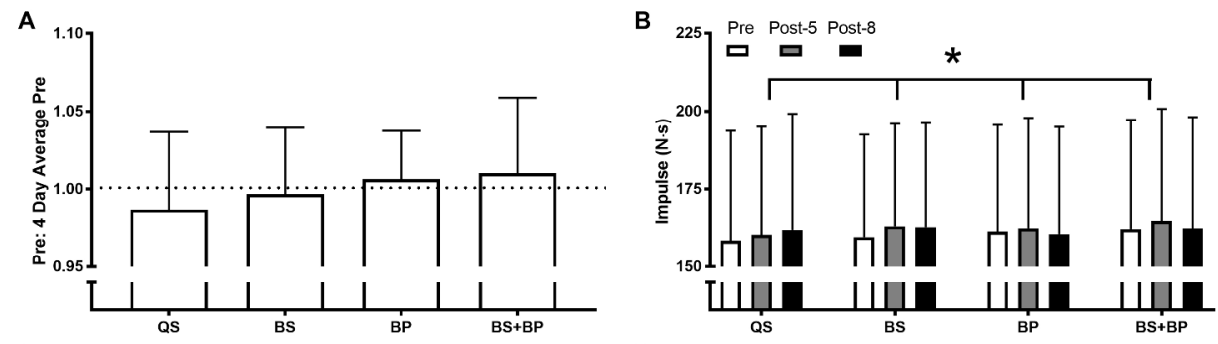

C
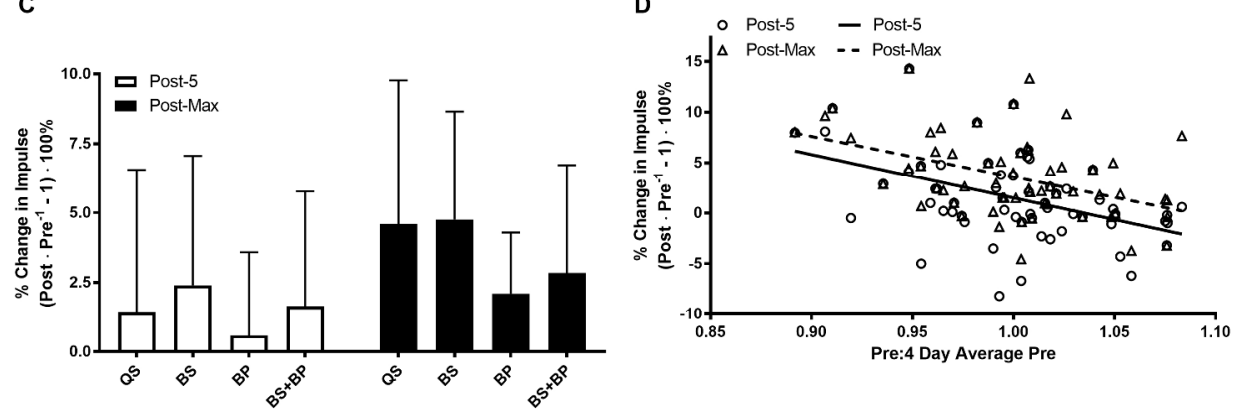

Figure 1: Squat jump performance during Pre and Post trials. A) Mean performance in Pre trials across the four test days (no significant differences). B) Squat jump impulse at Pre, Post-5 and Post 8 in the four test conditions. * $P<0.05$ vs Pre (Main effect of the 2 -way repeated measures ANOVA). C) Pre-Post changes in impulse at Post-5 and Post-Max for each conditioning activity (no significant differences). D) Scatter plot of Pre-Post change in impulse at Post- 5 and Post-Max versus the ratio of same-day Pre to the four day average of Pre values. Linear fits of the data have $r^{2}$ values of 0.20 for Post-5 and 0.19 for Post-Max. Both slopes are significantly different from zero with $P<0.001$. In $A-C$, values are presented as means $\pm S D ; n=15$. QS Quiet Standing; BS - Back Squat; BP - Bench Press.

$$
265 \times 164 \mathrm{~mm}(300 \times 300 \mathrm{DPI})
$$

